\title{
All finite sets are Ramsey in the maximum norm
}

\author{
Andrey Kupavskii ${ }^{1}$ and Arsenii Sagdeev ${ }^{2}$ \\ ${ }^{1}$ MIPT, Moscow, Russia; IAS, Princeton, USA; CNRS, Grenoble, France; E-mail: kupavskii@ya.ru. \\ ${ }^{2}$ MIPT, Moscow, Russia; E-mail: sagdeev.aa@phystech.edu.
}

Received: 4 August 2020; Accepted: 27 June 2021

2020 Mathematics Subject Classification: Primary - 05D10; Secondary - 52C10

\begin{abstract}
For two metric spaces $\mathbb{X}$ and $\mathcal{Y}$ the chromatic number $\chi(\mathbb{X} ; \mathcal{Y})$ of $\mathbb{X}$ with forbidden $\mathcal{Y}$ is the smallest $k$ such that there is a colouring of the points of $\mathbb{X}$ with $k$ colors that contains no monochromatic copy of $\mathcal{Y}$. In this article, we show that for each finite metric space $\mathcal{M}$ that contains at least two points the value $\chi\left(\mathbb{R}_{\infty}^{n} ; \mathcal{M}\right)$ grows exponentially with $n$. We also provide explicit lower and upper bounds for some special $\mathcal{M}$.
\end{abstract}

\section{Introduction}

Ramsey theory is a central part of modern combinatorics with many connections to other areas, such as logic, number theory and computer science. Its topic is to find homogeneous substructures in sufficiently large or dense structures. The early examples include Schur's lemma, van der Waerden's theorem and Ramsey's theorem. We refer the reader to the classical book [12] for a survey of early developments of Ramsey theory.

Most early results were concerned with finding homogeneous substructures in combinatorial structures (such as monochromatic complete subgraphs in any two-colouring of edges of a large complete graph). The problems we are interested in in this article are of geometric nature.

In 1950, Nelson posed the following question: What is the minimal number of colours needed to colour all points of the Euclidean plane $\mathbb{R}^{2}$ such that no two points at unit distance apart receive the same colour? This quantity is called the chromatic number of the plane and is denoted by $\chi\left(\mathbb{R}^{2}\right)$. This problem received great interest from the mathematics community, and even the origins of the questions spurred heated debates, partly because Nelson asked his question in private communication. We refer to the book of Soifer [33] and surveys of Raigorodskii [26, 28] for a comprehensive account of the problem.

For almost 70 years, the problem resisted attacks from numerous researchers, and the state of the art was at the easy-to-get lower and upper bounds $4 \leq \chi\left(\mathbb{R}^{2}\right) \leq 7$ due to Nelson and Isbell, respectively (or due to Mosers [20] and Hadwiger [13]; see the Soifer's historical research [33]). Recently, the combinatorics community was shaken when an amateur mathematician, de Grey [4], improved the lower bound to $\chi\left(\mathbb{R}^{2}\right) \geq 5$. A few weeks later, Exoo and Ismailescu [9] gave another proof of this bound. Since then, the initial de Grey's construction has been simplified several times within the framework of the Polymath16 Project (the current record is due to Parts [22]).

One natural generalisation of Nelson's problem concerns chromatic numbers of Euclidean spaces of other dimensions. One can find the current best lower and upper bounds on $\chi\left(\mathbb{R}^{n}\right)$ for several small values of $n$ in [1,3]. As for $n$ large, Frankl and Wilson [11] showed that the function $\chi\left(\mathbb{R}^{n}\right)$ grows exponentially with $n$. The best known asymptotic lower and upper bounds are $(1.239 \ldots+o(1))^{n} \leq$ 
$\chi\left(\mathbb{R}^{n}\right) \leq(3+o(1))^{n}$ as $n \rightarrow \infty$ (see Larman and Rogers [17] or Prosanov [23] for the upper bound and Raigorodskii [25] for the lower bound).

A systematic study of such questions on the interface of geometry and Ramsey theory, named Euclidean Ramsey theory, began with three papers [5, 6, 7] of Erdôs, Graham, Montgomery, Rothschild, Spencer and Straus. Given a subset $S \subset \mathbb{R}^{d}$ (with induced metric), the value $\chi\left(\mathbb{R}^{n} ; S\right)$ is defined to be the minimum number of colours needed to colour all points of Euclidean space $\mathbb{R}^{n}$ with no monochromatic isometric copy $S^{\prime} \subset \mathbb{R}^{n}$ of $S$. Note this this definition only makes sense if $|S| \geq 2$. In this case, the value $\chi\left(\mathbb{R}^{n} ; S\right)$ is well defined; that is, the corresponding minimum always exists, because we trivially have $\chi\left(\mathbb{R}^{n} ; S\right) \leq \chi\left(\mathbb{R}^{n}\right)$.

A set $S \subset \mathbb{R}^{d}$ is called $\ell_{2}-$ Ramsey if $\chi\left(\mathbb{R}^{n} ; S\right)$ tends to infinity as $n \rightarrow \infty$. Similarly, a set $S$ is called exponentially $\ell_{2}-$ Ramsey if there is a constant $\chi_{S}>1$ such that $\chi\left(\mathbb{R}^{n} ; S\right)>\left(\chi_{S}+o(1)\right)^{n}$ as $n \rightarrow \infty$. Relatively few sets are known to be exponentially $\ell_{2}-$ Ramsey. Frankl and Rödl [10] proved that the vertex sets of simplices and bricks (or hyperrectangles) are exponentially $\ell_{2}-$ Ramsey. One can find several explicit exponential lower and upper bounds for these sets in [21, 24, 29, 30, 31]. More sets are known to have a weaker property of being $\ell_{2}-$ Ramsey. Kř́íz [14] proved that each 'fairly symmetric' set is $\ell_{2}$-Ramsey. Later, this was used by him [15] and Cantwell [2] to show that the set of vertices of each regular polytope is $\ell_{2}$-Ramsey. Note that it is unknown whether there is an $\ell_{2}-$ Ramsey set that is not exponentially $\ell_{2}$-Ramsey.

At the same time, we know a strong necessary condition for a set to be $\ell_{2}$-Ramsey. Erdôs et al. showed that each $\ell_{2}$-Ramsey set must be finite [6] and spherical [5]; that is, be isometric to a subset of a sphere of some dimension. There is a popular conjecture stating that this is also sufficient. There is also a 'rival' conjecture proposed in [18] that states that only so-called subtransitive sets are $\ell_{2}$-Ramsey.

Another direction for generalisations that was explored is to work with other metrics than the Euclidean one. The natural candidates are the $\ell_{p}$-metrics, defined for $\mathbf{x}, \mathbf{y} \in \mathbb{R}^{n}$ by $\|\mathbf{x}-\mathbf{y}\|_{p}=$ $\left(\left|x_{1}-y_{1}\right|^{p}+\cdots+\left|x_{n}-y_{n}\right|^{p}\right)^{1 / p}$, or the Chebyshev metric (also known as the maximum metric) $\ell_{\infty}$ defined by $\|\mathbf{x}-\mathbf{y}\|_{\infty}=\max _{1 \leq i \leq n}\left\{\left|x_{i}-y_{i}\right|\right\}$. We denote the corresponding spaces by $\mathbb{R}_{p}^{n}$ and the corresponding chromatic numbers by $\chi\left(\mathbb{R}_{p}^{n}\right)$. For all $1<p<\infty, p \neq 2$, the best known lower bound due to Frankl and Wilson [11] is $\chi\left(\mathbb{R}_{p}^{n}\right) \geq(1.207 \ldots+o(1))^{n}$ as $n \rightarrow \infty$, whereas in case $p=1-$ that is, in case of the taxicab or Manhattan metric - Raigorodskii [27] proved a better lower bound $\chi\left(\mathbb{R}_{1}^{n}\right) \geq(1.366 \ldots+o(1))^{n}$. As for the upper bound, the best result for all real $p \neq 2$ due to the first author [16] is $\chi\left(\mathbb{R}_{p}^{n}\right) \leq(4+o(1))^{n}$ as $n \rightarrow \infty$ (also valid for any norm). The case $p=\infty$ stands out here because of the folklore equality $\chi\left(\mathbb{R}_{\infty}^{n}\right)=2^{n}$, valid for each $n \in \mathbb{N}$. For completeness, we will give its simple proof in the next section.

The questions mentioned above can be described using the following general setup. Let $\mathbb{X}=$ $\left(X, \rho_{X}\right), \mathcal{Y}=\left(Y, \rho_{Y}\right)$ be two metric spaces. A subset $Y^{\prime} \subset X$ is called a copy of $\mathcal{Y}$ if there is an isometry $f: Y \rightarrow Y^{\prime}$; that is, a bijection such that $\rho_{Y}\left(y_{1}, y_{2}\right)=\rho_{X}\left(f\left(y_{1}\right), f\left(y_{2}\right)\right)$ for all $y_{1}, y_{2} \in Y$. The chromatic number $\chi(\mathbb{X} ; \mathcal{Y})$ of the space $\mathbb{X}$ with a forbidden subspace $\mathcal{Y}$ is the minimal $k$ such that there is a colouring of elements of $X$ with $k$ colours and no monochromatic copy of $\mathcal{Y}$.

In this article, we focus on the case of $\mathbb{X}=\mathbb{R}_{\infty}^{n}$. Recall that for each finite metric space $\mathcal{M}$ of size at least 2 we trivially have $\chi\left(\mathbb{R}_{\infty}^{n} ; \mathcal{M}\right) \leq \chi\left(\mathbb{R}_{\infty}^{n}\right)=2^{n}$ for all $n \in \mathbb{N}$. As for the lower bound, observe that it is not even obvious that $\chi\left(\mathbb{R}_{\infty}^{n} ; \mathcal{M}\right)>1$ for some $n$. This inequality follows from a result due to Fréchet (see, e.g., [19]) that states that every finite metric space can be embedded into $\mathbb{R}_{\infty}^{n}$ for some $n \in \mathbb{N}$. We give the precise statement along with the short proof in Section 3 for completeness (see Lemma 2). The following theorem, which is the main result of our article, gives a much stronger lower bound.

Theorem 1. Any finite metric space $\mathcal{M}$ that contains at least two points is exponentially $\ell_{\infty}-$ Ramsey; that is, there is a constant $\chi_{\mathcal{M}}>1$ such that $\chi\left(\mathbb{R}_{\infty}^{n} ; \mathcal{M}\right)>\left(\chi_{\mathcal{M}}+o(1)\right)^{n}$ as $n \rightarrow \infty$.

The rest of the article is organised as follows. We start Section 2 by reciting the proof of $\chi\left(\mathbb{R}_{\infty}^{n}\right)=2^{n}$ and then prove Theorem 1 for ' 1 -dimensional' metric spaces, called batons. This step is crucial in the proof of Theorem 1, and the results in that section are also of independent interest. In Section 3 we 
deduce Theorem 1 from the results of Section 2. In Section 4 we provide upper bounds on the values $\chi\left(\mathbb{R}_{\infty}^{n} ; \mathcal{M}\right)$. Finally, Section 5 contains a discussion of the results and some open problems.

In what follows, whenever not specified, the distances are taken in the Chebyshev metric. We also slightly abuse notation and identify each set $S \subset \mathbb{R}^{d}$ with the corresponding metric space $\left(S, \ell_{\infty}\right)$, which is a subspace of $\mathbb{R}_{\infty}^{d}$.

\section{Batons}

For a $k \in \mathbb{N}$, denote $[k]_{0}=\{0,1,2, \ldots, k\}$ (note that this is a slightly nonstandard notation). Given a sequence of positive real numbers $\alpha_{1}, \ldots, \alpha_{k}$, a baton $\mathcal{B}\left(\alpha_{1}, \ldots, \alpha_{k}\right)$ is a metric space isometric to a set of points $\left\{0, \alpha_{1}, \alpha_{1}+\alpha_{2}, \ldots, \sum_{i=1}^{k} \alpha_{i}\right\} \subset \mathbb{R}$ with the metric induced from $\mathbb{R}$. If $\alpha_{1}=\cdots=\alpha_{k}=1$ then we denote this space $\mathcal{B}_{k}$ for shorthand.

\section{1. $\chi\left(\mathbb{R}_{\infty}^{n}\right)=2^{n}$}

We start the proofs with the simple but instructive case of $\chi\left(\mathbb{R}_{\infty}^{n}\right)=\chi\left(\mathbb{R}_{\infty}^{n}, \mathcal{B}_{1}\right)$. As we have already mentioned, the equality $\chi\left(\mathbb{R}_{\infty}^{n}\right)=2^{n}$ is folklore, and we give its proof for completeness.

Given $n \in \mathbb{N}$, let us denote $m=2^{n}$ for convenience. Let $\mathbf{v}_{1}, \ldots, \mathbf{v}_{m}$ be a set of vertices of a standard (discrete) unit cube $\{0,1\}^{n}$. Note that $\left\|\mathbf{v}_{i}-\mathbf{v}_{j}\right\|_{\infty}=1$ for all $i \neq j$, and thus $\chi\left(\mathbb{R}_{\infty}^{n}\right) \geq 2^{n}$ because we need to use a distinct colour for each $\mathbf{v}_{i}$.

To prove the matching upper bound, we explicitly describe the colouring. Let

$$
\mathcal{C}=\bigsqcup_{\mathbf{w} \in \mathbb{Z}^{n}}\left([0 ; 1)^{n}+2 \mathbf{w}\right)
$$

be a disjoint union of unit cubes. It is clear that for each $\mathbf{x}, \mathbf{y} \in \mathcal{C}$ we have $\|\mathbf{x}-\mathbf{y}\|_{\infty} \neq 1$. Indeed, one has $\|\mathbf{x}-\mathbf{y}\|_{\infty}<1$ whenever $\mathbf{x}$ and $\mathbf{y}$ come from the same unit cube, and $\|\mathbf{x}-\mathbf{y}\|_{\infty}>1$ whenever they are from different cubes. Given $i \leq m$, let us denote $\mathcal{C}_{i}=\mathcal{C}+\mathbf{v}_{i}$ (where $\mathbf{v}_{i}$ were defined in the previous paragraph). Colour each point of $\mathcal{C}_{i}$ with the $i$ th colour. We have $\bigsqcup_{i=1}^{m} \mathcal{C}_{i}=\mathbb{R}^{n}$, and thus it is a well-defined proper colouring of $\mathbb{R}^{n}$. This shows that $\chi\left(\mathbb{R}_{\infty}^{n}\right) \leq 2^{n}$.

\section{2. $\mathcal{B}_{k}$ is exponentially $\ell_{\infty}-$ Ramsey}

Theorem 2. Let $k, n$ be positive integers. Then each subset $X \subset[k]_{0}^{n} \subset \mathbb{R}_{\infty}^{n}$ of cardinality $|X|>k^{n}$ contains a copy of $\mathcal{B}_{k}$.

Proof. The proof is by induction on $n$. For $n=1$, there is nothing to prove. Indeed, if $X \subset[k]_{0}$ and $|X|>k$, then $X=[k]_{0}$ is the required copy of $\mathcal{B}_{k}$.

Next, assume that $n>1$. We employ a certain shifting-type argument. For a vector $\mathbf{x}=$ $\left(x_{1}, \ldots, x_{n}\right) \in[k]_{0}^{n}$ define its head $h(\mathbf{x})=x_{n}$ and tail $t(\mathbf{x})=\left(x_{1}, \ldots, x_{n-1}\right)$. Given $\mathbf{y} \in[k]_{0}^{n-1}$, let $H(\mathbf{y})=\left\{i \in[k]_{0}:(\mathbf{y}, i) \in X\right\}$. It should be clear that if $H(\mathbf{y})=[k]_{0}$ for some $\mathbf{y} \in[k]_{0}^{n-1}$, then a subset $\{(\mathbf{y}, 0),(\mathbf{y}, 1), \ldots,(\mathbf{y}, k)\} \subset X$ is the required copy of $\mathcal{B}_{k}$. In what follows, we assume that $H(\mathbf{y}) \neq[k]_{0}$ for all $\mathbf{y} \in[k]_{0}^{n-1}$.

Let us define a function $f: X \rightarrow[k]_{0}^{n}$ that increases the last coordinate of a vector by 1 'whenever possible' as follows. For a vector $\mathbf{x} \in X$,

$$
f(\mathbf{x})= \begin{cases}(t(\mathbf{x}), h(\mathbf{x})+1) & \text { if } \exists j \in[k]_{0} \backslash H(t(\mathbf{x})) \text { such that } j>h(\mathbf{x}) ; \\ \mathbf{x} & \text { otherwise. }\end{cases}
$$

We show that $f(\cdot)$ is an injection. Indeed, it is clear that $f\left(\mathbf{x}^{1}\right) \neq f\left(\mathbf{x}^{2}\right)$ for all $\mathbf{x}^{1}, \mathbf{x}^{2}$ such that $t\left(\mathbf{x}^{1}\right) \neq t\left(\mathbf{x}^{2}\right)$ or $\left|h\left(\mathbf{x}^{1}\right)-h\left(\mathbf{x}^{2}\right)\right| \geq 2$. Thus, let us consider $\mathbf{y} \in[k]_{0}^{n-1}, 0 \leq i<k$, such that both $(\mathbf{y}, i)$ and $(\mathbf{y}, i+1)$ belong to $X$. It is not hard to see that either $f((\mathbf{y}, i))=(\mathbf{y}, i+1), f((\mathbf{y}, i+1))=(\mathbf{y}, i+2)$ 
or $f((\mathbf{y}, i))=(\mathbf{y}, i), f((\mathbf{y}, i+1))=(\mathbf{y}, i+1)$. In both cases we have $f((\mathbf{y}, i)) \neq f((\mathbf{y}, i+1))$. Thus, $f(\cdot)$ is really an injection. Let $f(X)$ be the image of $X$ under $f(\cdot)$. Hence,

$$
|f(X)|=|X|>k^{n} .
$$

Partition $f(X)=X_{0} \sqcup \ldots \sqcup X_{k}$ based on the last coordinate:

$$
X_{i}=\{f(\mathbf{x}): \mathbf{x} \in X \text { and } h(f(\mathbf{x}))=i\} .
$$

Because $H(\mathbf{y}) \neq[k]_{0}$ for all $\mathbf{y} \in[k]_{0}^{n-1}$, it is easy to see that $X_{0}$ is empty. We conclude that

$$
|f(X)|=\left|X_{1}\right|+\cdots+\left|X_{k}\right| .
$$

It easily follows from comparing (1) and (2) that there is an $i \in\{1, \ldots, k\}$ such that $\left|X_{i}\right|>k^{n-1}$. Because distinct elements of $X_{i}$ have distinct tails, by the induction hypothesis one can find a set $\left\{\mathbf{y}^{0}, \ldots, \mathbf{y}^{k}\right\} \subset\left\{t(\mathbf{x}): \mathbf{x} \in X_{i}\right\}$ that forms a copy of $\mathcal{B}_{k}$. For each $j \in[k]_{0}, \operatorname{let} \mathbf{x}^{j}=f^{-1}\left(\mathbf{y}^{j}, i\right) \in X$ be the preimage of $\left(\mathbf{y}^{j}, i\right) \in X_{i}$. Note that we have $h\left(\mathbf{x}^{j}\right) \in\{i-1, i\}$ for each $j$, and thus $\left|h\left(\mathbf{x}^{j}\right)-h\left(\mathbf{x}^{j^{\prime}}\right)\right| \in\{0,1\}$ for all $j, j^{\prime} \in[k]_{0}$. This immediately implies that $\left\|\mathbf{x}^{j}-\mathbf{x}^{j^{\prime}}\right\|_{\infty}=\left\|\mathbf{y}^{j}-\mathbf{y}^{j^{\prime}}\right\|_{\infty}$ for all $j, j^{\prime} \in[k]_{0}$. Hence, the subset $\left\{\mathbf{x}^{0}, \ldots, \mathbf{x}^{k}\right\} \subset X$ is isometric to $\mathcal{B}_{k}$.

Theorem 2 implies that if the colouring of $\mathbb{R}_{\infty}^{n}$ contains no monochromatic copy of $\mathcal{B}_{k}$, then each of its colours can intersect $[k]_{0}^{n}$ in at most $k^{n}$ points. Using the pigeonhole principle, we get the following corollary.

Corollary 1. For each positive integer $k$, none has $\chi\left(\mathbb{R}_{\infty}^{n} ; \mathcal{B}_{k}\right) \geq\left(\frac{k+1}{k}\right)^{n}$.

\section{3. $\mathcal{B}(1, \alpha)$ is exponentially $\ell_{\infty}-$ Ramsey}

In this and the following two subsections we generalise Theorem 2 and Corollary 1 to the case of arbitrary batons. However, the proof in the general case is nontrivial, and we wanted to illustrate some of its ideas on a much simpler case of $\mathcal{B}(1, \alpha)$, which is a set $\{0,1,1+\alpha\}$ with the natural metric.

Theorem 3. Let $\alpha>1$ be a real number. Then there is a subset $A \subset \mathbb{R}$ of cardinality $\lceil\alpha\rceil+2$ such that the following holds. Given a positive integer $n$, each subset $B \subset A^{n} \subset \mathbb{R}_{\infty}^{n}$ of cardinality $|B|>(\lceil\alpha\rceil+1)^{n}$ contains a copy of $\mathcal{B}(1, \alpha)$.

Proof. Denote $m=\lceil\alpha\rceil$. Consider $A=\left\{a_{0}, \ldots, a_{m+1}\right\} \subset \mathbb{R}$, where $a_{l}$ are defined as follows:

$$
a_{0}=0, \quad a_{l}=1+\frac{l-1}{m-1}(\alpha-1) \text { for } 1 \leq l \leq m, a_{m+1}=\alpha+1
$$

In particular, $a_{1}=1, a_{m}=\alpha$. Define a bijection $f:[m+1]_{0}^{n} \rightarrow A^{n}$ by $f\left(\left(x_{1}, \ldots, x_{n}\right)\right)=$ $\left(a_{x_{1}}, \ldots, a_{x_{n}}\right)$.

Let $B \subset A^{n}$ be an arbitrary subset of cardinality $|B|>(m+1)^{n}$. From Theorem 2 it follows that there is a subset $\left\{\mathbf{x}^{0}, \ldots, \mathbf{x}^{m+1}\right\} \subset f^{-1}(B) \subset[m+1]_{0}^{n}$ that is a copy of $\mathcal{B}_{m+1}$. Without loss of generality, we can assume that $\left\|\mathbf{x}^{s}-\mathbf{x}^{t}\right\|_{\infty}=|s-t|$ for all $s, t \in[m+1]_{0}$. In particular, it is easy to see that $\left\{\mathbf{x}^{0}, \mathbf{x}^{1}, \mathbf{x}^{m+1}\right\}$ is a copy of $\mathcal{B}(1, m)$. For convenience, let us denote $\mathbf{x}^{0}, \mathbf{x}^{1}$ and $\mathbf{x}^{m+1}$ by $\mathbf{x}, \mathbf{y}$ and $\mathbf{z}$, respectively. Then the following two statements hold:

$$
\text { for all } i \in\{1, \ldots, n\} \text { one has }\left\{\begin{array}{l}
\left|x_{i}-y_{i}\right| \leq 1 \\
\left|y_{i}-z_{i}\right| \leq m
\end{array}\right.
$$


there is $j \in\{1, \ldots, n\}$ such that either $\left\{\begin{array}{l}x_{j}=0, \\ y_{j}=1, \\ z_{j}=m+1,\end{array}\right.$ or $\left\{\begin{array}{l}x_{j}=m+1, \\ y_{j}=m, \\ z_{j}=0 .\end{array}\right.$

The first statement is straightforward from the definition of $\mathcal{B}(1, m)$. As for the second, the equality $\|\mathbf{x}-\mathbf{z}\|_{\infty}=m+1$ implies that there is $j \in\{1, \ldots, n\}$ such that $\left|x_{j}-z_{j}\right|=m+1$, and thus either $x_{j}=0$ and $z_{j}=m+1$ or $x_{j}=m+1$ and $z_{j}=0$. Then it follows from (3) that $y_{j}=1$ in the former case and $y_{j}=m$ in the latter.

We claim that $\{f(\mathbf{x}), f(\mathbf{y}), f(\mathbf{z})\} \subset B$ is a copy of $\mathcal{B}(1, \alpha)$. To check that, we need to verify that the distances between $f(\mathbf{x}), f(\mathbf{y}), f(\mathbf{z})$ are the same as the distances between points in $\mathcal{B}(1, \alpha)$.

Clearly, $\left\|\mathbf{a}^{1}-\mathbf{a}^{2}\right\|_{\infty} \leq \alpha+1$ for all $\mathbf{a}^{1}, \mathbf{a}^{2} \in A^{n}$. At the same time, it follows from (4) that $\mid f(\mathbf{x})_{j}-$ $f(\mathbf{z})_{j}|=| a_{x_{j}}-a_{z_{j}} \mid=\alpha+1$, and thus $\|f(\mathbf{x})-f(\mathbf{z})\|_{\infty}=\alpha+1$.

Similarly, $\left|f(\mathbf{x})_{j}-f(\mathbf{y})_{j}\right|=1$ and $\left|f(\mathbf{y})_{j}-f(\mathbf{z})_{j}\right|=\alpha$, implying $\|f(\mathbf{x})-f(\mathbf{y})\|_{\infty} \geq 1$ and $\| f(\mathbf{y})-$ $f(\mathbf{z}) \|_{\infty} \geq \alpha$. We actually have equality in both of these inequalities. Let us show it for the former, and the latter is analogous. Indeed, if $\|f(\mathbf{x})-f(\mathbf{y})\|_{\infty}>1$, then there is $j^{\prime} \in\{1, \ldots, n\}$ such that $\left|f(\mathbf{x})_{j^{\prime}}-f(\mathbf{y})_{j^{\prime}}\right|>1$. It follows from the definition of $a_{l}$ that if $\left|a_{l}-a_{r}\right|>1$, then $|l-r| \geq 2$. Hence, $\left|x_{j^{\prime}}-y_{j^{\prime}}\right| \geq 2$, which contradicts (3).

Observe that now one can easily deduce from Theorem 3 that for all $\alpha>1$ and $n \in \mathbb{N}$, we have $\chi\left(\mathbb{R}_{\infty}^{n} ; \mathcal{B}(1, \alpha)\right) \geq\left(\frac{\lceil\alpha\rceil+2}{\lceil\alpha\rceil+1}\right)^{n}$. In particular, this implies that the metric space $\mathcal{B}(1, \alpha)$ is exponentially $\ell_{\infty}$-Ramsey.

\section{4. $\mathcal{B}\left(\alpha_{1}, \ldots, \alpha_{k}\right)$ is exponentially $\ell_{\infty}-$ Ramsey}

In this subsection we deal with the general case of $\mathcal{B}\left(\alpha_{1}, \ldots, \alpha_{k}\right)$. We use the same idea of reduction to the integer case and applying the pigeonhole principle. In the notation of the previous subsection, the main difficulty here is to find an appropriate $A$ and bijection $f$.

Theorem 4. Let $k$ be a positive integer and $\alpha_{1}, \ldots, \alpha_{k}$ be positive real numbers. Then there is an integer $m$ and a subset $A \subset \mathbb{R}$ of cardinality $m+1$ such that the following holds. Given a positive integer $n$, each subset $B \subset A^{n} \subset \mathbb{R}_{\infty}^{n}$ of cardinality $|B|>m^{n}$ contains a copy of $\mathcal{B}\left(\alpha_{1}, \ldots, \alpha_{k}\right)$.

First, we suppose that there exists an 'appropriate' finite subset $A=\left\{a_{0}, \ldots, a_{m}\right\} \subset \mathbb{R}$ that satisfies several conditions and deduce Theorem 4 using it. Then we prove the existence of this 'appropriate' $A$.

Lemma 1. Let $k$ be a positive integer and $\alpha_{1}, \ldots, \alpha_{k}$ be positive real numbers. Set

$$
\begin{aligned}
\Gamma=\{\gamma: \gamma & \leq \alpha_{1}+\cdots+\alpha_{k} \text { and } \\
\gamma & \left.=d_{1} \alpha_{1}+\cdots+d_{k} \alpha_{k} \text { for some } d_{1}, \ldots, d_{k} \in \mathbb{N} \cup\{0\}\right\} .
\end{aligned}
$$

Then there are positive integers $p_{1}, \ldots, p_{k}$ and a sequence of real numbers $a_{0}<\cdots<a_{m}$, where $m=p_{1}+\cdots+p_{k}$, such that the following two statements hold. First, for all positive integers $l$ and $r$ such that $l+r \leq m$, one has

$$
a_{l+r} \leq a_{l}+a_{r}
$$

Second, for each $\gamma=d_{1} \alpha_{1}+\cdots+d_{k} \alpha_{k} \in \Gamma$, one has $d_{1} p_{1}+\cdots+d_{k} p_{k} \leq m$ and

$$
a_{d_{1} p_{1}+\cdots+d_{k} p_{k}}=d_{1} \alpha_{1}+\cdots+d_{k} \alpha_{k} .
$$

Proof of Theorem 4. This proof essentially repeats the argument from the previous subsection. Take $p_{1}, \ldots, p_{k}, m$, and $a_{0}<\ldots<a_{m}$ as in Lemma 1. Set $A=\left\{a_{0}, \ldots, a_{m}\right\}$. Define a bijection 
$a:[m]_{0} \rightarrow A$ by $a(l)=a_{l}$ for all $l \in[m]_{0}$. This function is strictly increasing by construction. Given $n \geq 1$, let $f:[m]_{0}^{n} \rightarrow A^{n}$ be a bijection defined by $f\left(\left(x_{1}, \ldots, x_{n}\right)\right)=\left(a_{x_{1}}, \ldots, a_{x_{n}}\right)$. Note that we have $f(\mathbf{x})_{i}=a\left(x_{i}\right)=a_{x_{i}}$ for all $\mathbf{x}=\left(x_{1}, \ldots, x_{n}\right) \in[m]_{0}^{n}, 1 \leq i \leq n$.

Let $B \subset A^{n}$ be an arbitrary subset of cardinality $|B|>m^{n}$. From Theorem 2 it follows that there is a subset $\left\{\mathbf{x}^{0}, \ldots, \mathbf{x}^{m}\right\} \subset f^{-1}(B) \subset[m]_{0}^{n}$ that is a copy of $\mathcal{B}_{m}$. Without loss of generality, we can assume that $\left\|\mathbf{x}^{s}-\mathbf{x}^{t}\right\|_{\infty}=|s-t|$ for all $s, t \in[m]_{0}$. In particular, it is easy to see that $\left\{\mathbf{x}^{0}, \mathbf{x}^{p_{1}}, \mathbf{x}^{p_{1}+p_{2}}, \ldots, \mathbf{x}^{p_{1}+p_{2}+\cdots+p_{k}}\right\}$ is a copy of $\mathcal{B}\left(p_{1}, \ldots, p_{k}\right)$. For convenience, let us denote $\mathbf{x}^{0}, \mathbf{x}^{p_{1}}, \mathbf{x}^{p_{1}+p_{2}}, \ldots, \mathbf{x}^{p_{1}+p_{2}+\cdots+p_{k}}$ by $\mathbf{y}^{0}, \mathbf{y}^{1}, \ldots, \mathbf{y}^{k}$, respectively. They satisfy the following two properties:

for all $0 \leq s<t \leq k$ and for all $i \in\{1, \ldots, n\}$, one has

$$
\left|y_{i}^{s}-y_{i}^{t}\right| \leq p_{s+1}+\cdots+p_{t},
$$

there is $j \in\{1, \ldots, n\}$ such that

$$
\text { either }\left\{\begin{array} { l } 
{ y _ { j } ^ { 0 } = 0 , } \\
{ y _ { j } ^ { 1 } = p _ { 1 } , } \\
{ \cdots } \\
{ y _ { j } ^ { k } = p _ { 1 } + \cdots + p _ { k } , }
\end{array} \quad \text { or } \left\{\begin{array}{l}
y_{j}^{0}=p_{1}+\cdots+p_{k}, \\
y_{j}^{1}=p_{1}+\cdots+p_{k-1}, \\
\cdots \\
y_{j}^{k}=0 .
\end{array}\right.\right.
$$

The first property immediately follows from $\left\|\mathbf{y}^{s}-\mathbf{y}^{t}\right\|_{\infty}=p_{s+1}+\cdots+p_{t}$. As for the second, given that $\left\|\mathbf{y}^{0}-\mathbf{y}^{k}\right\|_{\infty}=m$ and $\mathbf{y}^{0}, \mathbf{y}^{k} \in[m]_{0}^{n}$, we must have a $j$ such that either $y_{j}^{0}=0$ and $y_{j}^{k}=m$ or $y_{j}^{0}=m$ and $y_{j}^{k}=0$. In the rest of this subsection we assume that the former holds (the other case is symmetric). Then for each $1 \leq s \leq k-1$, given the distances $\left\|\mathbf{y}^{0}-\mathbf{y}^{s}\right\|_{\infty}=p_{1}+\ldots+p_{s},\left\|\mathbf{y}^{s}-\mathbf{y}^{k}\right\|_{\infty}=p_{s+1}+\ldots+p_{k}=$ $m-\left\|\mathbf{y}^{0}-\mathbf{y}^{s}\right\|_{\infty}$, we clearly must have $y_{j}^{s}=p_{1}+\ldots+p_{s}$.

Using these two properties, we conclude the proof of Theorem 4 by showing that the set $\left\{f\left(\mathbf{y}^{0}\right), f\left(\mathbf{y}^{1}\right), \ldots, f\left(\mathbf{y}^{k}\right)\right\} \subset B$ is a copy of $\mathcal{B}\left(\alpha_{1}, \ldots, \alpha_{k}\right)$.

On the one hand, given $0 \leq s<t \leq k$, we use (6) and (8) to get that $f\left(\mathbf{y}^{s}\right)_{j}=\alpha_{1}+\ldots+\alpha_{s}$ and $f\left(\mathbf{y}^{t}\right)_{j}=\alpha_{1}+\ldots+\alpha_{t}$. This implies that

$$
\left|f\left(\mathbf{y}^{s}\right)_{j}-f\left(\mathbf{y}^{t}\right)_{j}\right|=\alpha_{s+1}+\ldots+\alpha_{t} .
$$

On the other hand, fix any $j^{\prime} \in\{1, \ldots, n\}$ and without loss of generality, assume that $y_{j^{\prime}}^{s} \geq y_{j^{\prime}}^{t}$. Using the monotonicity of $a(\cdot)$ and (5), we get that

$$
0 \leq f\left(\mathbf{y}^{s}\right)_{j^{\prime}}-f\left(\mathbf{y}^{t}\right)_{j^{\prime}}=a\left(y_{j^{\prime}}^{s}\right)-a\left(y_{j^{\prime}}^{t}\right)=a\left(y_{j^{\prime}}^{s}-y_{j^{\prime}}^{t}+y_{j^{\prime}}^{t}\right)-a\left(y_{j^{\prime}}^{t}\right) \leq a\left(y_{j^{\prime}}^{s}-y_{j^{\prime}}^{t}\right) .
$$

It follows from (7) that $y_{j^{\prime}}^{s}-y_{j^{\prime}}^{t} \leq p_{s+1}+\cdots+p_{t}$. Using the monotonicity of $a(\cdot)$ and (6), we get that

$$
a\left(y_{j^{\prime}}^{s}-y_{j^{\prime}}^{t}\right) \leq a\left(p_{s+1}+\cdots+p_{t}\right)=\alpha_{s+1}+\cdots+\alpha_{t} .
$$

We substitute (11) in (10) and get that $\left|f\left(\mathbf{y}^{s}\right)_{j^{\prime}}-f\left(\mathbf{y}^{t}\right)_{j^{\prime}}\right| \leq \alpha_{s+1}+\cdots+\alpha_{t}$. Together with (9) this gives $\left\|f\left(\mathbf{y}^{s}\right)-f\left(\mathbf{y}^{t}\right)\right\|_{\infty}=\alpha_{s+1}+\ldots+\alpha_{t}$ for all $0 \leq s<t \leq k$. This implies that $\left\{f\left(\mathbf{y}^{0}\right), f\left(\mathbf{y}^{1}\right), \ldots, f\left(\mathbf{y}^{k}\right)\right\} \subset$ $B$ is indeed a copy of $\mathcal{B}\left(\alpha_{1}, \ldots, \alpha_{k}\right)$.

It only remains to prove Lemma 1 in order to finish the proof of Theorem 4.

\subsection{The proof of Lemma 1}

Suppose that $\Gamma=\left\{\gamma_{0}, \ldots, \gamma_{t}\right\}$, where $\gamma_{0}<\cdots<\gamma_{t}$. In particular, $\gamma_{0}=0, \gamma_{1}=\min _{1 \leq i \leq k}\left\{\alpha_{i}\right\}$ and $\gamma_{t}=\alpha_{1}+\cdots+\alpha_{k}$. Let $\gamma_{t+1}$ be the smallest linear combination of $\alpha_{1}, \ldots, \alpha_{k}$ with nonnegative integer 
coefficients that is greater than $\gamma_{t}$; that is,

$$
\gamma_{t+1}=\min \left\{\gamma: \gamma>\gamma_{t} \text { and } \gamma=d_{1} \alpha_{1}+\cdots+d_{k} \alpha_{k} \text { for some } d_{1}, \ldots, d_{k} \in \mathbb{N} \cup\{0\}\right\} .
$$

Put $\delta=\min _{1 \leq i \leq t+1}\left\{\gamma_{i}-\gamma_{i-1}\right\}$ and $\theta=\gamma_{t} / \gamma_{1}$. Let $q_{0}$ be a large enough integer such that

$$
\frac{1}{q_{0}}<\delta \text { and } \frac{\theta}{q_{0}^{1+1 / k}}<\frac{1}{2 q_{0}} .
$$

We will apply the following result of Dirichlet on Diophantine approximations (see, e.g., [32], Section 2, Theorem 1A).

Theorem 5. Given $\alpha_{1}^{\prime}, \ldots, \alpha_{k}^{\prime} \in \mathbb{R}$ and $q_{0}^{\prime} \in \mathbb{N}$, there is an integer $q>q_{0}^{\prime}$ such that the following holds. There are $p_{1}, \ldots, p_{k} \in \mathbb{Z}$ such that for each $1 \leq i \leq k$, one has

$$
\left|\alpha_{i}^{\prime}-\frac{p_{i}}{q}\right|<\frac{1}{q^{1+1 / k}}
$$

Apply this theorem with $\alpha_{i}$ playing the role of $\alpha_{i}^{\prime}$ and $q_{0}$ playing the role of $q_{0}^{\prime}$. Let $q, p_{1}, \ldots, p_{k}$ be as in the conclusion of this theorem. Given $\gamma \in \mathbb{R}$, denote by $c(\gamma)=\lfloor q \gamma\rceil$ the numerator of the best rational approximation of $\gamma$ with denominator equal to $q$. Note that $\left|\gamma-\frac{c(\gamma)}{q}\right| \leq \frac{1}{2 q}$ for all $\gamma$. Moreover, if $\left|\gamma-\frac{c}{q}\right|<\frac{1}{2 q}$ for some $\gamma \in \mathbb{R}, c \in \mathbb{N}$, then $c=c(\gamma)$. The following two propositions show that the function $c(\cdot)$ is strictly increasing and 'linear' on $\Gamma$.

Proposition 1. One has $c\left(\gamma_{i}\right)>c\left(\gamma_{i-1}\right)$ for all $1 \leq i \leq t$.

Proof. Given $1 \leq i \leq t$, it is clear that $c\left(\gamma_{i}\right) \geq c\left(\gamma_{i-1}\right)$. Assume that $c\left(\gamma_{i}\right)=c\left(\gamma_{i-1}\right)$. Now it follows by the triangle inequality that

$$
\delta \leq\left|\gamma_{i}-\gamma_{i-1}\right| \leq\left|\gamma_{i}-\frac{c\left(\gamma_{i}\right)}{q}\right|+\left|\gamma_{i-1}-\frac{c\left(\gamma_{i-1}\right)}{q}\right| \leq \frac{1}{2 q}+\frac{1}{2 q}=\frac{1}{q}<\frac{1}{q_{0}},
$$

which contradicts the definition of $q_{0}$.

Proposition 2. Let $\gamma=d_{1} \alpha_{1}+\cdots+d_{k} \alpha_{k} \in \Gamma$. Then $c(\gamma)=d_{1} p_{1}+\cdots+d_{k} p_{k}$. In particular, for all $\gamma_{i}, \gamma_{i^{\prime}} \in \Gamma$ such that $\gamma_{i}+\gamma_{i^{\prime}} \in \Gamma$, one has $c\left(\gamma_{i}+\gamma_{i^{\prime}}\right)=c\left(\gamma_{i}\right)+c\left(\gamma_{i^{\prime}}\right)$.

Proof. Set $\varepsilon_{i}=\left|\alpha_{i}-\frac{p_{i}}{q}\right|$ and recall that $\varepsilon_{i} \leq q^{-(1+1 / k)}$. Recall that

$$
\theta=\frac{\gamma_{t}}{\gamma_{1}}=\frac{\alpha_{1}+\cdots+\alpha_{k}}{\min _{1 \leq i \leq k}\left\{\alpha_{i}\right\}}
$$

Because $\gamma \leq \alpha_{1}+\cdots+\alpha_{k}$, it is easy to see that $d_{1}+\cdots+d_{k} \leq \theta$. Now it is clear that

$$
\left|\gamma-\frac{d_{1} p_{1}+\cdots+d_{k} p_{k}}{q}\right| \leq d_{1} \varepsilon_{1}+\cdots+d_{k} \varepsilon_{k} \leq \frac{d_{1}+\cdots+d_{k}}{q^{1+1 / k}} \leq \frac{\theta}{q^{1+1 / k}}<\frac{1}{2 q},
$$

because $q>q_{0}$. This implies that $c(\gamma)=d_{1} p_{1}+\ldots+d_{k} p_{k}$. The second part of the proposition is immediate from the first part by the 'linearity' of $c(\gamma)$ on $\gamma \in \Gamma$.

Proposition 2 implies that $c\left(\gamma_{0}\right)=0$ and $c\left(\gamma_{t}\right)=p_{1}+\cdots+p_{k}=m$. We define the desired sequence $a_{0}, \ldots, a_{m}$ as follows. Set $a_{0}=0$. Given $0<l \leq m$, it follows from Proposition 1 that there is a unique $1 \leq i \leq t$ such that $c\left(\gamma_{i-1}\right)<l \leq c\left(\gamma_{i}\right)$. We set

$$
a_{l}=\gamma_{i}-\frac{c\left(\gamma_{i}\right)-l}{2 m} \delta
$$


To finish the proof of Lemma 1 we need to verify that this sequence is strictly increasing and satisfies (5) and (6).

First, we show that $a_{l}>a_{l-1}$ for all $1 \leq l \leq m$. If there is $1 \leq i \leq t$ such that $c\left(\gamma_{i-1}\right)<l-1<l \leq$ $c\left(\gamma_{i}\right)$, then $a_{l}-a_{l-1}=\frac{1}{2 m} \delta>0$. Moreover, for all $1 \leq i \leq t$, one has

$$
a_{c\left(\gamma_{i-1}\right)+1}-a_{c\left(\gamma_{i-1}\right)}=\left(\gamma_{i}-\frac{c\left(\gamma_{i}\right)-c\left(\gamma_{i-1}\right)-1}{2 m} \delta\right)-\gamma_{i-1}>\left(\gamma_{i}-\gamma_{i-1}\right)-\frac{\delta}{2} \geq \frac{\delta}{2}>0 .
$$

Second, for all $0 \leq i \leq t$ we have $a_{c\left(\gamma_{i}\right)}=\gamma_{i}$ by construction. Therefore, Proposition 2 implies that the sequence $a_{0}, \ldots, a_{m}$ satisfies (6).

Finally, given $0 \leq l, r \leq m$ such that $l+r \leq m$, we need to check that $a_{l+r} \leq a_{l}+a_{r}$. Observe that there is nothing to check if either $l=0$ or $r=0$. Thus, without loss of generality, we assume that both $l$ and $r$ are greater than 0 . Under this assumption there are unique $1 \leq i, i^{\prime}, j \leq t$ such that $c\left(\gamma_{i-1}\right)<l \leq c\left(\gamma_{i}\right), c\left(\gamma_{i^{\prime}-1}\right)<r \leq c\left(\gamma_{i^{\prime}}\right)$ and $c\left(\gamma_{j-1}\right)<l+r \leq c\left(\gamma_{j}\right)$. Set $\gamma=\gamma_{i}+\gamma_{i^{\prime}}$.

Assume that $\gamma<\gamma_{j}$. Then $\gamma \in \Gamma$ and we can apply Proposition 2 to get that $c\left(\gamma_{j-1}\right)<l+r \leq$ $c\left(\gamma_{i}\right)+c\left(\gamma_{i^{\prime}}\right)=c(\gamma)$. Thus, by Proposition 1 we get that $\gamma>\gamma_{j-1}$ and so $\gamma \geq \gamma_{j}$, a contradiction. Thus, $\gamma \geq \gamma_{j}$

Suppose that $\gamma=\gamma_{j}$. Then Proposition 2 implies that $c(\gamma)=c\left(\gamma_{i}\right)+c\left(\gamma_{i^{\prime}}\right)$, and we have

$$
\begin{aligned}
a_{l}+a_{r}-a_{l+r} & =\left(\gamma_{i}-\frac{c\left(\gamma_{i}\right)-l}{2 m} \delta\right)+\left(\gamma_{i^{\prime}}-\frac{c\left(\gamma_{i^{\prime}}\right)-r}{2 m} \delta\right)-\left(\gamma_{j}-\frac{c\left(\gamma_{j}\right)-l-r}{2 m} \delta\right) \\
& =\left(\gamma_{i}+\gamma_{i^{\prime}}-\gamma\right)+\frac{c(\gamma)-c\left(\gamma_{i}\right)-c\left(\gamma_{i^{\prime}}\right)}{2 m} \delta=0 .
\end{aligned}
$$

Suppose that $\gamma>\gamma_{j}$ and, consequently, $\gamma \geq \gamma_{j+1}$. The sequence of $a_{i}$ s is increasing, and so $a_{l+r} \leq a_{c\left(\gamma_{j}\right)}=\gamma_{j}$. We conclude that

$$
\begin{aligned}
a_{l}+a_{r}-a_{l+r} & \geq\left(\gamma_{i}-\frac{c\left(\gamma_{i}\right)-l}{2 m} \delta\right)+\left(\gamma_{i^{\prime}}-\frac{c\left(\gamma_{i^{\prime}}\right)-r}{2 m} \delta\right)-\gamma_{j} \\
& >\left(\gamma_{i}+\gamma_{i^{\prime}}-\gamma_{j}\right)-\frac{\delta}{2}-\frac{\delta}{2} \geq\left(\gamma_{j+1}-\gamma_{j}\right)-\delta \geq 0 .
\end{aligned}
$$

This concludes the proof of Lemma 1.

\section{The proof of Theorem 1}

We begin with some notation that was introduced (in a slightly different form) by Frankl and Rödl [10]. Given a real $p \geq 1$ or $p=\infty$, a metric space $\mathcal{M}$ is called $\ell_{p}$ - super-Ramsey (with parameters $F_{\mathcal{M}}$ and $\left.\chi_{\mathcal{M}}\right)$ if there exist constants $F_{\mathcal{M}} \geq \chi_{\mathcal{M}}>1$ and a sequence of sets $V_{\mathcal{M}}(n) \subset \mathbb{R}_{p}^{n}$ such that $\left|V_{\mathcal{M}}(n)\right| \leq\left(F_{\mathcal{M}}+o(1)\right)^{n}$ and each subset of $V_{\mathcal{M}}(n)$ of size greater than $\left|V_{\mathcal{M}}(n)\right|\left(\chi_{\mathcal{M}}+o(1)\right)^{-n}$, $n \rightarrow \infty$, contains a copy of $\mathcal{M}$. An easy application of the pigeonhole principle as in Corollary 1 shows that for each $\ell_{p}$-super-Ramsey metric space $\mathcal{M}$ with parameters $F_{\mathcal{M}}$ and $\chi_{\mathcal{M}}$ that contains at least two points, one has $\chi\left(\mathbb{R}_{p}^{n} ; \mathcal{M}\right) \geq(\chi \mathcal{M}+o(1))^{n}$ as $n \rightarrow \infty$. So, the $\ell_{p}$-super-Ramsey property implies the exponentially $\ell_{p}$-Ramsey one. Therefore, in order to prove Theorem 1 it is sufficient to prove the following statement.

Theorem 6. Any finite metric space is $\ell_{\infty}$-super-Ramsey.

Given a real $p \geq 1$ and given two metric spaces $\mathcal{X}=\left(X, \rho_{X}\right)$ and $\mathcal{Y}=\left(Y, \rho_{Y}\right)$, their Cartesian product $\mathcal{X} \times \mathcal{Y}$ is the metric space $(X \times Y, \rho)$, where

$$
\rho\left(\left(x_{1}, y_{1}\right),\left(x_{2}, y_{2}\right)\right)=\left(\rho_{X}\left(x_{1}, x_{2}\right)^{p}+\rho_{Y}\left(y_{1}, y_{2}\right)^{p}\right)^{1 / p}
$$


for all $x_{1}, x_{2} \in X$ and $y_{1}, y_{2} \in Y$. In a special case $p=\infty$, to which we devote the present article, we set

$$
\rho\left(\left(x_{1}, y_{1}\right),\left(x_{2}, y_{2}\right)\right)=\max \left\{\rho_{X}\left(x_{1}, x_{2}\right), \rho_{Y}\left(y_{1}, y_{2}\right)\right\} .
$$

Frankl and Rödl [10] showed that a Cartesian product of any two $\ell_{2}$-super-Ramsey finite metric spaces is also $\ell_{2}$-super-Ramsey. Their proof actually works for the $\ell_{p}$ metric for all $p$. In case $p=\infty$, the proof is spelled out in a paper by the second author [29], where he also gives an explicit dependence of the parameters.

Theorem 7 ([10], Theorem 2.2; [29], Proposition 1.). Let $\mathcal{X}$ and $\mathcal{Y}$ be $\ell_{\infty}$-super-Ramsey finite metric spaces. Then $\mathcal{X} \times \mathcal{Y}$ is also $\ell_{\infty}$-super-Ramsey.

Note that Theorem 4 implies that each baton $\mathcal{B}$ is $\ell_{\infty}$-super-Ramsey (with the parameters $F_{\mathcal{B}}=m+1$ and $\chi_{\mathcal{B}}=\frac{m+1}{m}$, where $m$ is from Theorem 4). Thus, by Theorem 7 , we conclude that for all $d \in \mathbb{N}$, each $d$-dimensional grid - that is, a Cartesian product of $d$ batons - is also $\ell_{\infty}$-super-Ramsey. It should be clear that the property of being $\ell_{\infty}$-super-Ramsey (with parameters $F$ and $\chi$ ) is hereditary with respect to taking subsets. Because each finite subset $\left\{\mathbf{x}^{0}, \ldots, \mathbf{x}^{k}\right\} \subset \mathbb{R}^{d}$ is a subset of the $d$-dimensional grid $\prod_{i=1}^{d}\left\{x_{i}^{0}, \ldots, x_{i}^{k}\right\}$, we get the following corollary.

Corollary 2. Given $d \in \mathbb{N}$, any finite $S \subset \mathbb{R}_{\infty}^{d}$ is $\ell_{\infty}$-super-Ramsey.

The following simple proposition due to Fréchet (see [19]) concludes the proof of Theorem 6.

Lemma 2. Any metric space $\mathcal{M}=(M, \rho)$ with $|M|=d$ is isometric to a subset $S \subset \mathbb{R}_{\infty}^{d}$.

Proof. Let $M=\left\{u_{1}, \ldots, u_{d}\right\}$. The desired set $S$ is given by the rows of the distance matrix $D=$ $\left(\rho\left(u_{i}, u_{j}\right)\right)_{i, j=1}^{d}$.

\section{Upper bounds}

Let $\mathcal{M}=\left(M, \rho_{M}\right)$ be a finite metric space that contains at least two points. Theorem 1 guarantees the existence of an exponential lower bound on $\chi\left(\mathbb{R}_{\infty}^{n} ; \mathcal{M}\right)$. Recall that we also have $\chi\left(\mathbb{R}_{\infty}^{n} ; \mathcal{M}\right) \leq \chi\left(\mathbb{R}_{\infty}^{n}\right)=$ $2^{n}$. In this section we give a better upper bound. Essentially the same proof appeared in [24], [23].

For a metric space $\mathcal{M}$, let $d(\mathcal{M})$ be the diameter of $\mathcal{M}$; that is, the maximum over distances between pair of points of $\mathcal{M}$. Let $l(\mathcal{M})>0$ be the smallest number such that for each two points $x, y \in M$ there are $z_{0}, z_{1}, \ldots, z_{t} \in M$ such that $z_{0}=x, z_{t}=y$, and $\rho_{M}\left(z_{i}, z_{i+1}\right) \leq l$ for all $0 \leq i \leq t-1$.

In what follows, we denote the natural logarithm by $\log (\cdot)$.

Theorem 8. For each finite metric space $\mathcal{M}$ that contains at least two points, one has

$$
\chi\left(\mathbb{R}_{\infty}^{n} ; \mathcal{M}\right)<(1+o(1)) n \log n\left(1+\frac{l(\mathcal{M})}{d(\mathcal{M})}\right)^{n}
$$

as $n \rightarrow \infty$.

Proof. Fix arbitrary positive $d^{\prime}<d(\mathcal{M})$ and $l^{\prime}>l(\mathcal{M})$. Let $C=\left[0 ; d^{\prime}\right]^{n}$ be an $n$-dimensional cube and put $\mathcal{C}=\bigsqcup_{\mathbf{v} \in \mathbb{Z}^{n}}\left(C+\left(d^{\prime}+l^{\prime}\right) \mathbf{v}\right) \subset \mathbb{R}^{n}$.

Assume that there is a subset $M^{\prime} \subset \mathcal{C}$ that is a copy of $\mathcal{M}$ and consider any $\mathbf{x}, \mathbf{y} \in M^{\prime}$. Let $\mathbf{z}_{0}, \mathbf{z}_{1}, \ldots, \mathbf{z}_{t} \in M^{\prime}$ be a sequence of points of $M^{\prime}$ such that $\mathbf{z}_{0}=\mathbf{x}, \mathbf{z}_{t}=\mathbf{y}$ and $\left\|\mathbf{z}_{i}-\mathbf{z}_{i+1}\right\|_{\infty} \leq l(M)$ for all $0 \leq i \leq t-1$. Because the distance between any two different translates of $C$ in $\mathcal{C}$ is at least $l^{\prime}>l(\mathcal{M})$, we conclude that $\mathbf{z}_{i}$ and $\mathbf{z}_{i+1}$ belong to the same translate of $C$ for all $0 \leq i \leq t-1$. Hence, $\mathbf{x}$ and $\mathbf{y}$ belong to the same translate of $C$, and thus $M^{\prime}$ lies entirely within the same translate of $C$. However, this is impossible, because the diameter of $C$ is equal to $d^{\prime}<d(\mathcal{M})$. Thus, $\mathcal{C}$ does not contain a copy of $\mathcal{M}$.

To conclude the proof, we use the classical Erdôs-Rogers result [8], which states that $(1+$ $o(1)) n \log n\left(1+\frac{l^{\prime}}{d^{\prime}}\right)^{n}$ translates of $\mathcal{C}$ are sufficient to cover $\mathbb{R}^{n}$. Colour all points of the $i$ th translate of $\mathcal{C}$ from the covering in one colour. This is clearly a valid colouring. We also remark that $o(1)$ in the last 
formula does not depend on $d^{\prime}$ and $l^{\prime}$. Hence, letting $d^{\prime} \rightarrow d$ and $l^{\prime} \rightarrow l$, we obtain the claimed upper bound.

It is straightforward from the definition that $l(\mathcal{M}) \leq d(\mathcal{M})$ for all metric spaces $\mathcal{M}$. Note that the upper bound from Theorem 8 is slightly worse than the trivial bound $\chi\left(\mathbb{R}_{\infty}^{n} ; \mathcal{M}\right) \leq 2^{n}$ if $l(\mathcal{M})=d(\mathcal{M})$ but is asymptotically better if $l(\mathcal{M})<d(\mathcal{M})$.

Let $\mathcal{M}$ be a metric space such that $l(\mathcal{M})<d(\mathcal{M})$ and $\frac{l(\mathcal{M})}{d(\mathcal{M})} \in \mathbb{Q}$. Applying a proper homothety, one can assume without loss of generality that $l(\mathcal{M}), d(\mathcal{M}) \in \mathbb{N}$. We can slightly improve the result of Theorem 8 for such metric spaces using a simpler probabilistic argument.

Theorem 9. Let $\mathcal{M}=\left(M, \rho_{M}\right)$ be a finite metric space such that $|M| \geq 2, l(\mathcal{M})$ and $d(\mathcal{M})$ are integers satisfying $l(\mathcal{M})<d(\mathcal{M})$. Then, we have

$$
\chi\left(\mathbb{R}_{\infty}^{n} ; \mathcal{M}\right)<(1+o(1)) n \log d(\mathcal{M})\left(1+\frac{l(\mathcal{M})}{d(\mathcal{M})}\right)^{n}
$$

as $n \rightarrow \infty$.

Proof. For shorthand, denote $l=l(\mathcal{M}), d=d(\mathcal{M})$ and $m=d+l \in \mathbb{N}$. Let $C=[0 ; d)^{n}$ and $\mathcal{C}=\bigsqcup_{\mathbf{v} \in \mathbb{Z}^{n}}(C+m \mathbf{v}) \subset \mathbb{R}^{n}$. As in the proof of Theorem 8, one can see that $\mathcal{C}$ does not contain a copy of $\mathcal{M}$, and we can use the same colour for all of its points. It only remains to cover $\mathbb{R}^{n}$ using as few translates of $\mathcal{C}$ as possible.

Set $s=\left\lfloor n \log d\left(\frac{m}{d}\right)^{n}\right\rfloor$ and let $\mathbf{v}_{1}, \ldots, \mathbf{v}_{s}$ be the elements of $[m-1]_{0}^{n}$ chosen uniformly and independently at random. Let $X \subset[m-1]_{0}^{n}$ be the subset consisting of all points $\mathbf{x} \in[m-1]_{0}^{n}$ that do not belong to any $\mathcal{C}+\mathbf{v}_{i}$, where $1 \leq i \leq s$.

It is easy to see that for $\mathbf{x} \in[m-1]_{0}^{n}$ and $1 \leq i \leq s$, we have $\operatorname{Pr}\left[\mathbf{x} \in \mathcal{C}+\mathbf{v}_{i}\right]=\left(\frac{d}{m}\right)^{n}$. Hence, from the mutual independence of the choice of $\mathbf{v}_{i}$ s it follows that

$$
\operatorname{Pr}[\mathbf{x} \in X]=\left(1-\left(\frac{d}{m}\right)^{n}\right)^{s}
$$

The linearity of the expectation gives

$$
\mathrm{E}[|X|]=\left(1-\left(\frac{d}{m}\right)^{n}\right)^{s} m^{n}<\left(1-\left(\frac{d}{m}\right)^{n}\right)^{n \log d\left(\frac{m}{d}\right)^{n}} m^{n}<e^{-n \log d} m^{n}=\left(\frac{m}{d}\right)^{n} .
$$

Thus, there is a way to fix the choice of $\mathbf{v}_{1}, \ldots, \mathbf{v}_{s} \in[m-1]_{0}^{n}$ such that $|X| \leq\left(\frac{m}{d}\right)^{n}$. Consider the translates $\left\{\mathcal{C}+\mathbf{v}_{i}: 1 \leq i \leq s\right\} \cup\{\mathcal{C}+\mathbf{x}: \mathbf{x} \in X\}$. Together, they cover all points of $[m-1]_{0}^{n}$ by construction. Moreover, they cover all points of $\mathbb{R}^{n}$ by periodicity. Finally, the number of translates we used is equal to $s+|X|=(1+o(1)) n \log d\left(\frac{m}{d}\right)^{n}$ as required.

\section{Concluding remarks and open problems}

One of the key objects in this article are the batons $\mathcal{B}_{k}$. It follows from Corollary 1 and Theorem 9 that $\chi\left(\mathbb{R}_{\infty}^{n} ; \mathcal{B}_{k}\right)=\left(\frac{k+1}{k}+o(1)\right)^{n}$ as $n \rightarrow \infty$. However, by calculating the exact values of $\chi\left(\mathbb{R}_{\infty}^{n} ; \mathcal{B}_{k}\right)$ for some pairs of fixed small $n$ and $k$, we found that both the lower and upper bounds are not tight.

For other batons the situation is much worse. For instance, given $\alpha>1$, it follows from Theorems 3 and 8 that $\left(\frac{\lceil\alpha\rceil+2}{\lceil\alpha\rceil+1}\right)^{n} \leq \chi\left(\mathbb{R}_{\infty}^{n} ; \mathcal{B}(1, \alpha)\right) \leq\left(1+\frac{\alpha}{1+\alpha}+o(1)\right)^{n}$. Neither of these two bounds appears to be tight in general, and it is an interesting problem to determine the correct base of the exponent for this function.

For metric spaces other than batons, Theorem 1 does not immediately give an explicit exponential lower bound. One may, of course, directly follow its proof to extract the lower bound on $\chi_{\mathcal{M}}>1$ for 
each specific $\mathcal{M}$. The resulting bound would strongly depend on the dimension of the grid in which we embed $\mathcal{M}$, because each application of Theorem 7 weakens the lower bound significantly. For example, a lower bound for 2-dimensional grid $\mathcal{B}_{2}^{2}=\mathcal{B}_{2} \times \mathcal{B}_{2}$ one can extract from our proof is only $\chi\left(\mathbb{R}_{\infty}^{n} ; \mathcal{B}_{2}^{2}\right) \geq(1.0667 \ldots+o(1))^{n}$. We managed to generalise the argument from Section 2 to make it applicable not only for batons but also for different multidimensional grids. One of the results that we are able to obtain is that for each fixed $k$ and $m$, one has $\chi\left(\mathbb{R}_{\infty}^{n} ; \mathcal{B}_{k}^{m}\right)=\left(\frac{k+1}{k}+o(1)\right)^{n}$ as $n \rightarrow \infty$; that is, the base of the exponent does not depend on $m$. This result will be one of the subjects of a separate paper that is currently in preparation.

Finally, let us explicitly state a question that arises in connection with the covering technique from Section 4.

Problem 1. What is the smallest number $c(n)$ of translates of cubes $\{0,1\}^{n}$ that is needed to cover the torus $\mathbb{Z}_{3}^{n}$ ?

The best upper and lower bounds that we know of are $C_{1}(3 / 2)^{n} \leq c(n) \leq C_{2} n(3 / 2)^{n}$ for some constants $C_{1}, C_{2}$. Although the problem might appear simple, it seems to contain the difficulties that one is typically faced with when working with coverings as in Section 4.

Acknowledgments. We thank A.M. Raigorodskii for his helpful suggestions and active interest in our work during the preparation of this article. We also thank the referee for their helpful comments.

\section{Conflict of Interest: None.}

Financial Support. The authors acknowledge the financial support from the Ministry of Education and Science of the Russian Federation in the framework of MegaGrant No. 075-15-2019-1926, the Russian Foundation for Basic Research Grant No. 2031-70039 and No. 20-31-90009 and the Council for the Support of Leading Scientific Schools of the President of the Russian Federation (Grant No. N.Sh.-2540.2020.1). The research of the second author was supported in part by the Simons Foundation and by the Moebius Contest Foundation for Young Scientists. The second author is a Young Russian Mathematics award winner and would like to thank its sponsors and jury.

\section{References}

[1] L. I. Bogoliubsky and A. M. Raigorodskii, 'A Remark on lower bounds for the chromatic numbers of spaces of small dimension with metrics $\ell_{1}, \ell_{2}$ ', Math. Notes. 105 (2019), 180-203.

[2] K. Cantwell, All regular polytopes are Ramsey, J. Combin. Theory Ser. A, 114 (2007), 555-562.

[3] D. Cherkashin, A. Kulikov and A. Raigorodskii, 'On the chromatic numbers of small-dimensional Euclidean spaces', Discrete Appl. Math. 243 (2018), 125-131.

[4] A. D. N. J. de Grey, 'The chromatic number of the plane is at least 5', Geombinatorics 28 (2018), $18-31$.

[5] P. Erdős, R. L. Graham, P. Montgomery, B. L. Rothschild, J. Spencer and E. G. Straus, 'Euclidean Ramsey theorems I', J. Combin. Theory Ser. A 14 (1973), 341-363.

[6] P. Erdős, R. L. Graham, P. Montgomery, B. L. Rothschild, J. Spencer and E. G. Straus, 'Euclidean Ramsey theorems II', in Infinite and Finite Sets I (North Holland, Amsterdam, 1975), 529-557.

[7] P. Erdős, R. L. Graham, P. Montgomery, B. L. Rothschild, J. Spencer and E.G. Straus, 'Euclidean Ramsey theorems III, in' Infinite and Finite Sets (North-Holland, Amsterdam, 1973), 559-583.

[8] P. Erdős and C. A. Rogers, 'Covering space with convex bodies', Acta Arith. 7 (1962), 281-285.

[9] G. Exoo and D. Ismailescu, 'The chromatic number of the plane is at least 5: a new proof', Discrete Comput. Geom. 64 (2020), 216-226.

[10] P. Frankl and V. Rödl, 'A partition property of simplices in Euclidean space', J. Amer. Math. Soc. 3 (1990), 1-7.

[11] P. Frankl and R. M. Wilson, 'Intersection theorems with geometric consequences', Combinatorica 1 (1981), 357-368.

[12] R. L. Graham, B. L. Rothschild and J. H. Spencer, Ramsey theory, in Wiley-Intersci. Ser. Discrete Math. Optim., 2nd ed. (John Wiley \& Sons, New York, 1990).

[13] H. Hadwiger, 'Uberdeckung des euklidischen Raum durch kongruente Mengen, Portugaliae', Math. 4 (1945), $238-242$.

[14] I. Kř́žz, 'Permutation groups in Euclidean Ramsey theory', Proc. Amer. Math. Soc. 112 (1991), 899-907.

[15] I. Kř́̌̌z, 'All trapezoids are Ramsey', Discrete Math. 108 (1992), 59-62.

[16] A. Kupavskiy, 'On the chromatic number of $\mathbb{R}^{n}$ with an arbitrary norm', Discrete Math., 311 (2011), 437-440.

[17] D. G. Larman and C. A. Rogers, 'The realization of distances within sets in Euclidean space', Mathematika 19 (1972), 1-24.

[18] I. Leader, P. A. Russell and M. Walters, 'Transitive sets in Euclidean Ramsey theory', J. Combin. Theory Ser. A 119 (2012), 382-396. 
[19] J. Matoušek, Lectures on Discrete Geometry (Springer New York, 2002).

[20] L. Moser and W. Moser, 'Solution to problem 10', Can. Math. Bull. 4 (1961), 187-189.

[21] E. Naslund, 'Monochromatic Equilateral Triangles in the Unit Distance Graph', Bulletin of the London Mathematical Society, 52 (2020), 687-692.

[22] J. Parts, 'Graph minimization, focusing on the example of 5-chromatic unit-distance graphs in the plane', (2020), https://arxiv.org/abs/2010.12665.

[23] R. Prosanov, 'A new proof of the Larman-Rogers upper bound for the chromatic number of the Euclidean space', Discrete Appl. Math. 276 (2020), 115-120.

[24] R. I. Prosanov, 'Upper bounds for the chromatic numbers of Euclidean spaces with forbidden Ramsey sets', Math. Notes 103 (2018), 243-250.

[25] A. M. Raigorodskii, 'On the chromatic number of a space', Russian Math. Surveys 55 (2000), 351-352.

[26] A. M. Raigorodskii, 'The Borsuk problem and the chromatic numbers of some metric spaces', Russian Math. Surveys 56 (2001), 103-139.

[27] A. M. Raigorodskii, 'On the chromatic number of a space with the metric $\ell_{q}$ ', Russian Math. Surveys 59 (2004), 973 - 975.

[28] A. M. Raigorodskii, Coloring Distance Graphs and Graphs of Diameters, Thirty Essays on Geometric Graph Theory (Springer-Verlag New York, 2013), 429-460.

[29] A. A. Sagdeev, 'Exponentially Ramsey sets', Problems of Information Transmission 54 (2018), 372-396.

[30] A. A. Sagdeev, 'On a Frankl-Wilson theorem', Problems of Information Transmission 55 (2019), 376-395.

[31] A. A. Sagdeev and A. M. Raigorodskii, 'On a Frankl-Wilson theorem and its geometric corollaries', Acta Math. Univ. Comenianae 88 (2019), 1029-1033.

[32] W. M. Schmidt, Diophantine Approximation (Springer, Berlin, Heidelberg, 1996).

[33] A. Soifer, The Mathematical Coloring Book: Mathematics of Coloring and the Colorful Life of Its Creators (Springer-Verlag New York, 2008). 